DOI 10. 18307/2017. 0303

(c) 2017 by Journal of Lake Sciences

\title{
巢湖藻类高斯垂向分布结构参数的遥感估算”
}

\author{
梁其椿 ${ }^{1,2}$, 张玉超 $^{1,3 * *}$,薛 坤 ${ }^{1,2}, \mathbf{E}^{\text {洪涛 }}{ }^{1}$, 马荣华 ${ }^{1,3}$ \\ (1: 中国科学院南京地理与湖泊研究所, 中国科学院流域地理学重点实验室, 南京 210008) \\ (2: 中国科学院大学,北京 100049) \\ (3: 淮阴师范学院,江苏省区域现代农业与环境保护协同创新中心,淮安 223001)
}

摘 要: 藻类垂向分布异质性导致了遥感反演的湖泊表层叶绿素 $\mathrm{a}$ 浓度结果与单元水柱内藻类生物量间不存在一一对 应的关系, 因此有效确定藻类垂向分布结构是遥感反演湖泊藻类生物量的基础. 受自身因素和外环境条件的影响, 藻类 垂向分布结构呈现出多种类型,其中高斯类型应用最广. 本文基于 3200 组 HydroLight 模拟的高斯垂向数据构建 BP 神经 网络, 实现用 MODIS 数据相对应的 3 个波段的遥感反射比 $R_{\mathrm{rs}}(469) 、 R_{\mathrm{rs}}(555) 、 R_{\mathrm{rs}}(645)$ 和表层叶绿素 $\mathrm{a}$ 浓度共同估算高 斯垂向分布结构参数 $h$ 和 $\sigma$. 经巢湖地面实测数据验证显示, $h$ 和 $\sigma$ 的估算值与实测值的相关系数分别为 0.97 和 0.95 , 对 应的相对误差分别为 $13.20 \%$ 和 $12.36 \%$,两者相对误差同时小于 $30 \%$ 的占总数据量的 $87.5 \%$,表明该 BP 神经网络估算巢 湖藻类高斯垂向分布结构的有效性和准确性, 为基于卫星遥感数据获取湖泊藻类生物量提供了重要的理论基础.

关键词: 叶绿素 $\mathrm{a}$; 高斯垂向分布;HydroLight;BP 神经网络;巢湖

\section{Remote sensing-based estimation for Gaussian distribution parameters of vertical structure of algal biomass in Lake Chaohu}

\author{
LIANG Qichun $^{1,2}$, ZHANG Yuchao ${ }^{1,3 * *}$, XUE Kun ${ }^{1,2}$, DUAN Hongtao ${ }^{1}$ \& MA Ronghua ${ }^{1,3}$ \\ (1: Key Laboratory of Watershed Geographic Sciences, Nanjing Institute of Geography and Limnology, Chinese Academy of \\ Sciences, Nanjing 210008, P.R.China) \\ (2: University of Chinese Academy of Sciences, Beijing 100049, P.R.China) \\ ( 3: Jiangsu Collaborative Innovation Center of Regional Modern Agriculture \& Environmental Protection, Huaiyin Normal Uni- \\ versity, Huaian 223001, P.R.China)
}

Abstract: The relationship between water surface reflectance and total depth integrated algae biomass can be very complex as different kinds of algal vertical distributions can occur. For this reason, effectively identifying the algae vertical profiles is fundamental to estimate algal biomass. Gaussian profiles are the most typical algae vertical profiles which occur in most environmental conditions (including external and internal system). In this research, a back propagation (BP) neural network was established to estimate Gaussian distribution parameters of the vertical structure $h$ and $\sigma$ by wave bands $R_{\mathrm{rs}}(469), R_{\mathrm{rs}}(555), R_{\mathrm{rs}}(645)$ and chlorophyll-a concentration band $C_{\text {Chl.a }}(0)$. The BP neural network was trained by using 3000 simulated datasets (radiative transfer simulation based on in-situ measured data by HydroLight), and verified by another 200 groups of simulated data and measured data. The correlation coefficient between estimated and measured $h$ and $\sigma$ were 0.97 and 0.95 , while the relative errors were $13.20 \%$ and $12.36 \%$, respectively. The relative error of $h$ and $\sigma$ was mostly less than $30 \%$. This indicated that it is a good effectiveness of BP neural networks to estimate the vertical distribution parameters and able to explore the three dimensional algal distribution in Lake Chaohu, thereby providing a significant theoretical basis for remote sensing estimation of algal biomass.

Keywords: Chlorophyll-a; Gaussian vertical distribution; HydroLight; back propagation neural network; Lake Chaohu

* 国家自然科学基金项目 (41431176,41471287) 和江苏省区域现代农业与环境保护协同创新中心面上项目 (HSXT236) 联合资助. 2016-04-21 收稿;2016-07-18 收修改稿. 梁其椿( 1991 ) , 男, 硕士研究生; E-mail: lqc_ niglas@163.com.

** 通信作者; E-mail:yczhang@ niglas.ac.cn. 
湖泊水色遥感可以利用多种星载传感器探测以及反演内陆水体叶绿素等水体水色要素参数 ${ }^{[1]}$. 遥感器 接收到的水体遥感反射比是光线经由水面射人水体, 经过水体以及水体中各组分的吸收和散射共同作用 后, 射出水面的离水辐亮度与总人射辐照度之比,包含了水体中一定深度内垂向各水层的光学特性信息 ${ }^{[2]}$. 传统的湖泊水色遥感大多基于水色参数或水体光学特性在垂向上是均质的假设,但事实上由于气象、环境 条件以及藻颗粒自身因素的影响, 湖泊水体中藻类垂向分布具有明显的不均一性 ${ }^{[3]}$. 藻类垂向分布的不均 一性导致了表层叶绿素 a ( Chl.a ) 浓度反演结果与单元水柱内藻类生物量间不存在一一对应关系,所以有效 确定叶绿素 a 浓度的垂向分布是遥感反演藻类生物量的基础.

目前针对藻类垂向分布的研究主要集中在大洋 I 类水体, 受生活习性和外环境条件影响, 藻类垂向分布 结构呈现出多种类型: 高斯分布 ${ }^{[4]}$ 、指数分布 ${ }^{[5]}$ 、线性分布 ${ }^{[6]}$ 等, 其中高斯分布应用最广泛. 国内外学者通过 研究 I 类水体中藻类高斯垂向分布对遥感反射比的影响, 基于统计回归 ${ }^{[7-8]}$ 、人工神经网络 ${ }^{[9-10]}$ 等方法成功 获取高斯垂向分布结构参数. 与大洋水体相比, 富营养化湖泊水深较浅, 在风、浪以及藻类自身生理特征影 响下 ${ }^{[11-12]}$, 湖泊藻类垂向呈现多样化、多变性的特点. 巢湖是我国典型的内陆富营养化湖泊 ${ }^{[13]}$, Xue 等 ${ }^{[14]}$ 针 对巢湖藻类垂向分布野外实测, 通过最优曲线拟合的方法将巢湖藻类垂向分布归结为 4 种类型:垂向均一 分布、高斯分布、指数分布、幂函数分布. 马孟采等 ${ }^{[15]}$ 也有类似结论, 且强调高斯模型分布是水面未发生藻 华时叶绿素 $\mathrm{a}$ 的主要垂向分布形式. 因此, 研究藻类高斯垂向分布结构参数的遥感估算, 是实现获取富营养 化湖泊藻总量的关键环节. 此外, 富营养化湖泊水体光学活性物质组成较为复杂, 其光学特性不仅仅受浮游 植物的影响 ${ }^{[16]}$, 这进一步加大了湖泊藻类垂向分布结构参数估算的难度和精度.

本文根据巢湖野外观测数据和同步外环境数据, 利用 HydroLight 开展了巢湖水体辐射传输模拟, 构建藻 类高斯垂向分布结构参数 $\left(c_{0} 、 h\right.$ 和 $\left.\sigma\right)$ 与遥感反射比 $\left(R_{\mathrm{rs}}\right)$ 映射关系的模拟数据库; 在分析高斯垂向分布结 构参数对 $R_{\mathrm{rs}}$ 影响的基础上,选取 MODIS 数据的对应波段 469、555 和 $645 \mathrm{~nm}$ 处的 $R_{\mathrm{rs}}$ 和表层叶绿素 a 浓度 $C_{\text {Chl. }}(0)$ 作为输人参数, 构建了估算藻类高斯垂向结构参数 $c_{0} 、 h$ 和 $\sigma$ 的 BP 神经网络估算模型; 利用模拟数 据和实测数据对模型的估算精度开展了测试与验证; 最后针对 HydroLight 辐射传输模拟的参数设定、藻类高 斯垂向分布结构的影响因素以及卫星数据的推广应用进行了讨论. 本研究工作是富营养化湖泊藻总量遥感 估算的重要基础, 可为湖泊蓝藻水华生态灾害监测和预测预警提供重要的技术支撑.

\section{1 材料与方法}

\section{1 研究区域概况}

巢湖 ( $31^{\circ} 25^{\prime} 28^{\prime \prime} \sim 31^{\circ} 43^{\prime} 28^{\prime \prime} \mathrm{N}, 117^{\circ} 16^{\prime} 54^{\prime \prime} \sim 117^{\circ} 51^{\prime} 46^{\prime \prime} \mathrm{E}$ ), 是长江水系下游湖泊, 位于安徽省中部, 由合 肥市、巢湖市、肥东县、肥西县、庐江县二市三县环抱, 是我国五大淡水湖之一, 是合肥市和巢湖市最重要的 生活水源 ${ }^{[17]}$. 巢湖平均水深约 $2.96 \mathrm{~m}$, 湖水面积约 $770 \mathrm{~km}^{2}$, 年内水位变幅大, 透明度低, 湖滨带水生植物分 布较少 ${ }^{[18]}$. 巢湖人湖河流约 33 条, 其中主要出人河流有 9 条. 过去 30 年中, 由于城市污水和工业污水等外 源污染的注人,巢湖的水污染和富营养化问题越来越严重 ${ }^{[19-20]}$, 暴发了多次蓝藻水华 ${ }^{[21]}$.

1.1.1 野外数据采集与分析 分别于 2013 年 5 月 28 日、7 月 24 日和 2015 年 5 月 24 日开展巢湖野外采样, 采样点数分别为 $9 、 2 、 15$ 个(图 1), 每个采样点垂向采集水样的水深分别为 $0 、 0.1 、 0.2 、 0.4 、 0.7 、 1.0 、 1.5 、 2.0$ 、 $3.0 \mathrm{~m}$, 采样时天空晴朗, 无云或者少云. 现场测量每个点位的 $R_{\mathrm{rs}}$ 、水深、透明度、风速、风向等, 并且每个采样 点于 9 个水深处各采集 $500 \mathrm{ml}$ 水样, 用于测定叶绿素 a、有色可溶解性有机物 (CDOM)、溶解态有机碳 (DOC) 浓度等.

1.1.2 叶绿素 $\mathrm{a}$ 浓度测定与处理 水样经过 GF/C 膜过滤后, 通过冻融萃取, 以 $90 \%$ 丙酮溶液做参比, 利用分 光光度计测定叶绿素 $\mathrm{a}$ 浓度 ${ }^{[22]}$.

1.1.3 水体光谱测量与处理 利用美国 ASD 公司生产的 FieldSpec Pro Dual VNIR 野外双通道光谱仪,采用水 面以上测量法获取水体 $350 \sim 1050 \mathrm{~nm}$ 范围内波段的遥感反射光谱 ${ }^{[23]}$.

在避开太阳直射反射、忽略或避开水面泡沫的情况下, 水体总光谱信号 $\left(L_{\mathrm{sw}}\right)$ 可近似看做水体离水辐亮 度 $\left(L_{\mathrm{w}}\right)$ 和天空光辐亮度 $\left(L_{\mathrm{sky}}\right)$ 之和, 即:

$$
L_{\mathrm{sw}}=L_{\mathrm{w}}+r \cdot L_{\mathrm{sky}}
$$




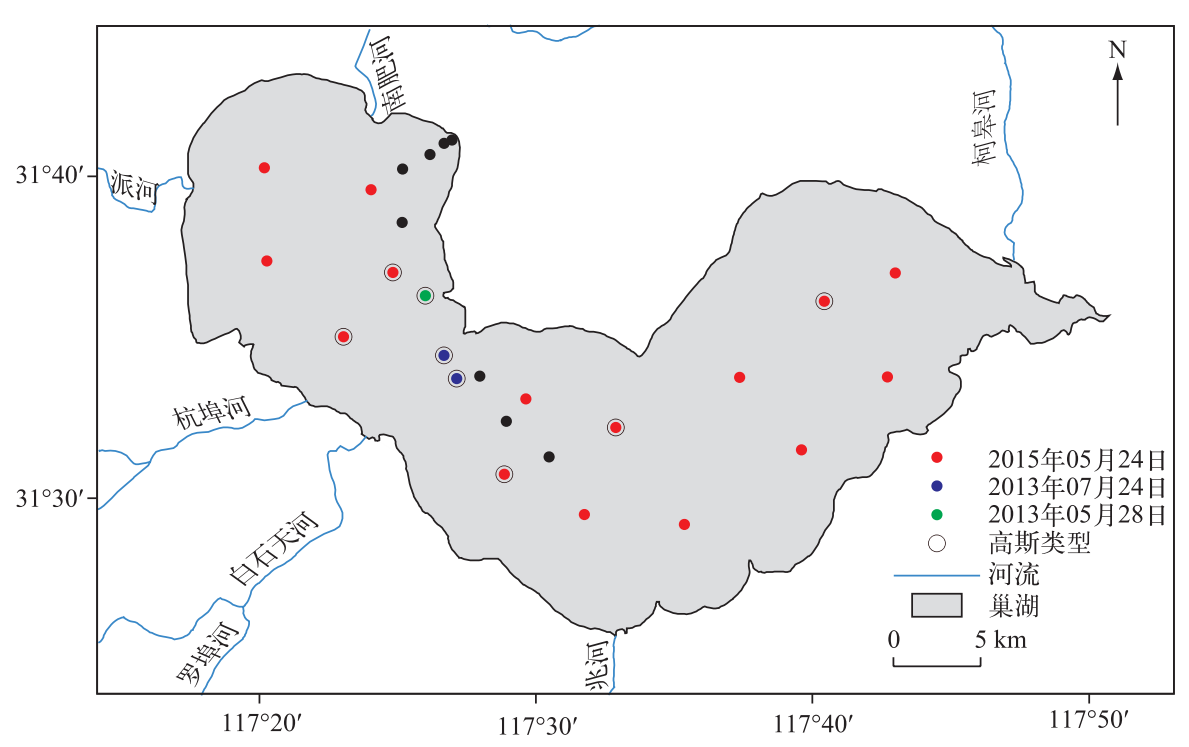

图 1 采样点分布

Fig.1 Distribution of sampling sites

式中, $r$ 为气一水界面对天空光的反射率, 与风速、太阳高度角和观测几何等有关, 取值为 $0.026 \sim 0.028$.

由于 $L_{\mathrm{w}}$ 在遥感中容易测定, 因此, 遥感反射比 $\left(R_{\mathrm{rs}}, \mathrm{sr}^{-1}\right)$ 可以定义为水体离水辐亮度与恰好位于水体表 面的向下辐照度 $\left(E_{\mathrm{d}}\left(0^{+}\right)\right)$的比值, 即:

$$
R_{\mathrm{rs}}=\frac{L_{\mathrm{w}}}{E_{\mathrm{d}}\left(0^{+}\right)}
$$

$E_{\mathrm{d}}\left(0^{+}\right)$可表示为:

$$
E_{\mathrm{d}}\left(0^{+}\right)=\frac{\pi \cdot L_{\mathrm{p}}}{\rho_{\mathrm{p}}}
$$

式中, $L_{\mathrm{p}}$ 为标准白板辐亮度, $\rho_{\mathrm{p}}$ 为标准白板的反射率, 校正值为 $30 \%$.

根据以上各式可以推导出水体遥感反射比为:

\section{2 藻类高斯垂向分布的数学表达}

$$
R_{\mathrm{rs}}=\frac{\rho_{\mathrm{p}}\left(L_{\mathrm{sw}}-r \cdot L_{\mathrm{sky}}\right)}{\pi \cdot L_{\mathrm{p}}}
$$

野外实测数据表明, 巢湖藻类垂向呈高斯分布时, 叶绿素 $\mathrm{a}$ 浓度最大值出现在水体表层 (深度 $\approx 0 \mathrm{~m}$ ), 与已有研究结果一致 ${ }^{[4]}$, 叶绿素 $\mathrm{a}$ 浓度垂向分布的高斯模型公式为 ${ }^{[14]}$ :

$$
C_{\text {Chl.a }}(z)=c_{0}+\frac{h}{\sigma \sqrt{2 \pi}} \exp \left[-\frac{1}{2}\left(\frac{z}{\sigma}\right)^{2}\right]
$$

式中, $C_{\text {Chl. }}(z)$ 是深度 $z$ 处的叶绿素 a 浓度; $c_{0}$ 是背景叶绿素 a 浓度值 $(\mu \mathrm{g} / \mathrm{L}) ; h$ 是与峰值强度有关的参数; $\sigma$ 是标准偏差, 与分层中叶绿素极大值宽度有关; $z$ 是水深 $(\mathrm{m})$.

\section{3 野外观测数据集}

对 26 个采样点的叶绿素 $\mathrm{a}$ 垂向分布数据 (每个采样点分别测量 9 个不同水深 $(0 、 0.1 、 0.2 、 0.4 、 0.7 、 1.0$ 、 $1.5 、 2.0 、 3.0 \mathrm{~m}$ ) 的叶绿素 $\mathrm{a}$ 浓度) 分别进行曲线拟合 (线性函数、二次多项式、高斯函数、指数函数、幂指数), 把具有最大 $R^{2}(>0.85)$ 、最小 $R M S E$ 的函数类型作为该采样点的最优垂向分布类型 ${ }^{[14]}$, 结果显示, 8 组叶绿 素 $\mathrm{a}$ 垂向分布数据的最优垂向分布类型为高斯分布类型, 本研究以此 8 组高斯类型垂向数据作为神经网络 模型的实测验证数据( 分布情况见图 1). 


\section{4 基于野外观测数据构建 HydroLight 模拟数据库}

HydroLight 是根据文献 [24]用 Fortran 语言编写的辐射传输 模型. 本文基于 HydroLight 的 CASE 2 模型计算了无机悬浮物 (SPIM) 和 CDOM 吸收光谱形态因子 $a_{g}(440)^{[25]}$ 不变的条件下, 与叶绿素 a 浓度垂向分布结构相关的固有光学性质 (IOPs) 的垂 直分布对 $R_{\mathrm{rs}}$ 的影响. 模拟的光谱范围是 $400 \sim 700 \mathrm{~nm}$, 间隔 5 $\mathrm{nm}$. 叶绿素 a 浓度垂向分布以巢湖野外观测数据为依据, $c_{0} 、 h$ 和 $\sigma$ 的设定范围分别为 2 40、5 80 和 $0.1 \sim 1$, 覆盖了野外观测 数据的分布范围, 又考虑到模拟数据库的运行效率,分别设定间 隔为 $2 、 5$ 和 0.1 ,共 3200 组. 其他 HydroLight 辐射传输模拟的输 人参数均取 2014 年巢湖对应平均值,具体见表 1 .

\section{BP 神经网络模型的设计}

在 $c_{0} 、 h$ 和 $\sigma$ 已知的条件下,即可获取叶绿素 a 浓度的垂向分布. 内陆湖泊光学组分复杂,且存在大量光 学浅水区 ${ }^{[16]}, c_{0}$ 情况复杂多变, 而 $C_{\text {Chl.a }}(0)$ 可以通过遥感反演, 所以本文选择 $h$ 和 $\sigma$ 为神经网络反演对象, 结合 $C_{\text {Chl. }}(0)$, 获取 $c_{0}$ 值.

\section{1 基于光谱特征确定神经网络的输入参量}

如图 2 所示, $R_{\mathrm{rs}}$ 呈现了典型的湖泊水体可见光光谱特征: 由于叶绿素 a 在蓝紫光波段的吸收峰以及黄 色物质的吸收作用, 在 400 500 $\mathrm{nm}$ 范围内 $R_{\mathrm{rs}}$ 较低 ${ }^{[26]} ; 580 \mathrm{~nm}$ 波长附近属于叶绿素 a 和胡萝卜素弱吸收区 域,加上细胞散射作用的影响, 所以该波长附近出现反射峰 ${ }^{[27]} ; 630 \mathrm{~nm}$ 附近呈肩状或反射谷, 原因是藻蓝素 在此处的吸收峰, 以及叶绿素 a 等有机质反射能力在 $600 \mathrm{~nm}$ 后逐步下降 ${ }^{[28]}$; 由于叶绿素 a 在 $682 \mathrm{~nm}$ 附近 的强烈吸收作用,该波段附近出现反射谷 ${ }^{[29]}$.

图 2 中 3 条垂线分别对应着 MODIS 的 band $3 、 4 、 1$ 的中心波长. Pearson 相关性分析表明, $\sigma$ 与 $R_{\mathrm{rs}}(469) 、 R_{\mathrm{rs}}(555)$ 和 $R_{\mathrm{rs}}(645)$ 呈现显著正相 关 $(r$ 分别为 $0.584 、 0.519 、 0.47, P<0.01)$, 而 $h$ 与 $R_{\mathrm{rs}}(469) 、 R_{\mathrm{rs}}(555)$ 和 $R_{\mathrm{rs}}(645)$ 则呈现显著负相 关 $(r$ 分别为 $-0.635 、-0.668 、-0.681, P<0.01)$.

基于上述光谱特征分析,并考虑卫星的波段 设置, 本研究选取 $R_{\mathrm{rs}}(469) 、 R_{\mathrm{rs}}(555) 、 R_{\mathrm{rs}}(645)$ 和 $C_{\text {Chl.a }}(0)$ 作为神经网络模型的输人参量.

\subsection{BP 神经网络隐含层结构设计}

应用 $\mathrm{BP}$ 神经网络需要事先确定好网络结 构,网络结构优化的重点和难点就是设计隐含层

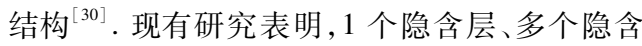
层节点的 3 层 BP 神经网络能有效逼近任何归 一化的输人一输出映射 ${ }^{[31]}$. 因此, 本研究也采用 1 个隐含层的 $\mathrm{BP}$ 神经网络. 为了获取最佳隐含 层节点数, 本研究构造若干不同隐含层节点的网 络分别进行训练, 选择性能最好的网络 ${ }^{[32]}$.

\subsection{BP 神经网络研究方案}

$1)$ 选取 $R_{\mathrm{rs}}(469) 、 R_{\mathrm{rs}}(555) 、 R_{\mathrm{rs}}(645)$ 和 $C_{\mathrm{Chl.a}}(0)$ 作为神经网络模型的输人参量, 输出参量是 $h$ 和 $\sigma$;

2) 确定 BP 神经网络的结构: 1 个隐含层,多个隐含层节点;

3) 从模拟数据库中随机挑选 3000 组数据作为训练数据, 剩下的 200 组作为测试数据, 8 组实测数据为
表 1 基于野外实测数据的 HydroLight 辐射传输模拟输入参数

Tab.1 Input parameters of HydroLight radiative transfer simulation based on field data

\begin{tabular}{cc}
\hline 参数 & 设定值 \\
\hline 太阳天顶角 $/{ }^{\circ}$ & 30 \\
风速 $/(\mathrm{m} / \mathrm{s})$ & 2.25 \\
水体折射率 & 1.34 \\
$\mathrm{SPIM} /(\mathrm{mg} / \mathrm{L})$ & 35.45 \\
$a_{\mathrm{g}}(440) / \mathrm{m}^{-1}$ & 0.6 \\
光谱斜率 $S_{\mathrm{g}} / \mathrm{nm}^{-1}$ & 0.019 \\
\hline
\end{tabular}

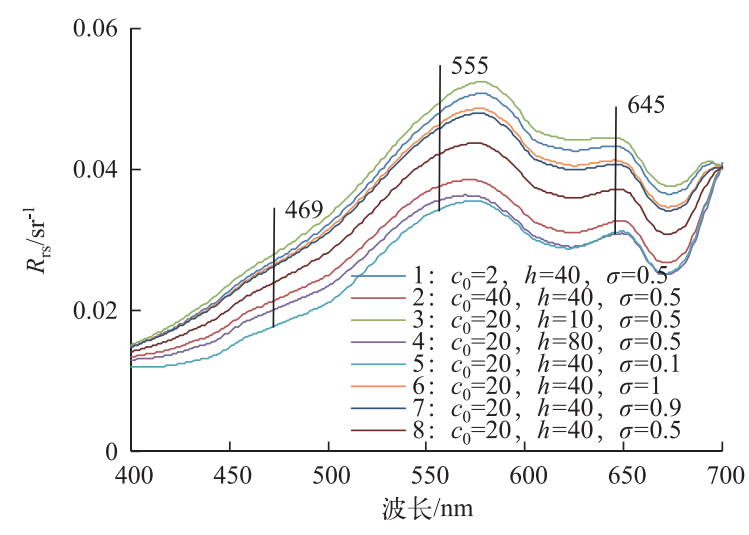

图 $2 c_{0} 、 h$ 和 $\sigma$ 参数一个变化其他不变 得到的代表性光谱曲线对比图

Fig. 2 The comparison chart of representative spectral curve corresponding to several chlorophyll-a concentrations Gaussian model 
验证数据;

4)采用 Matlab 的 newff 函数构建 BP 神经网络, BP 神经网络的计算流程见图 3.

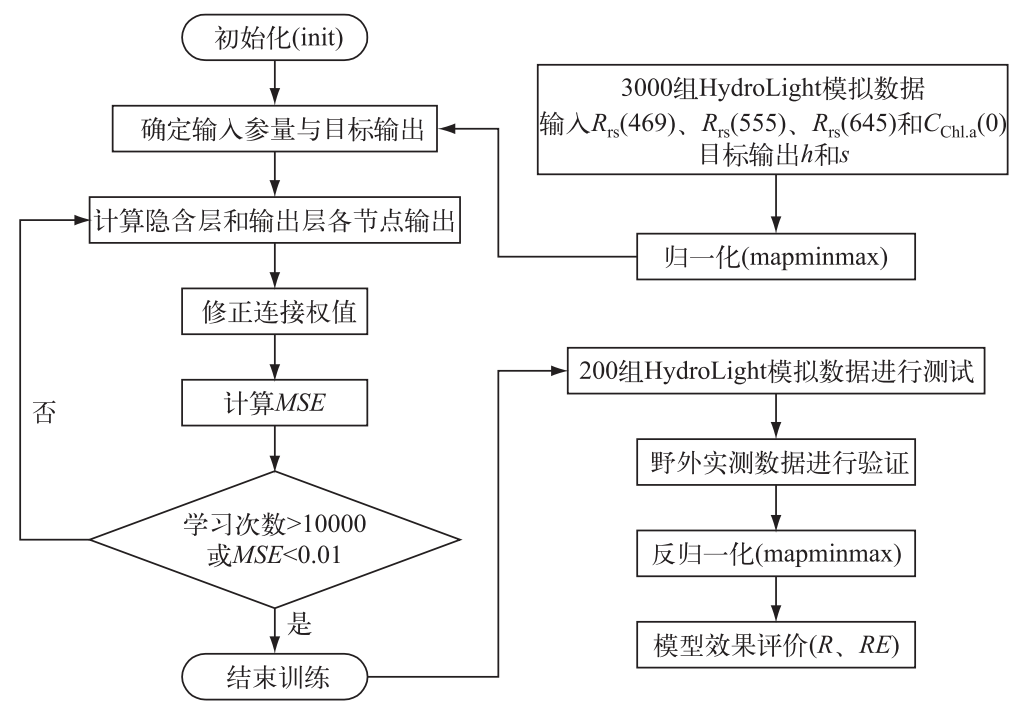

图 $3 \mathrm{BP}$ 神经网络的计算流程

Fig.3 Flow chart of BP neural network

\section{4 评价指标}

采用 Matlab 构建 BP 神经网络默认的训练性能 (performance) 评价指标为均方误差 (Mean Square Error, $M S E)$ :

$$
M S E=\frac{\sum_{i=1}^{n}\left(\text { predicted }_{i}-\text { target }_{i}\right)^{2}}{n}
$$

网络估算结果评价采用相关系数 $(R)$ 和相对误差 $(R E), R$ 和 $R E$ 计算公式分别如下:

$$
\begin{gathered}
R=\frac{\sum_{i=1}^{n}\left(\text { test }_{i}-\overline{\text { test }^{\prime}}\right)\left(\text { predicted }_{i}-\overline{\text { predicted }}\right)}{\sqrt{\sum_{i=1}^{n}\left(\text { test }_{i}-\overline{\text { test }^{2}}\right)^{2} \cdot \sum_{i=1}^{n}\left(\text { predicted }_{i}-\overline{\text { predicted }}\right)^{2}}} \\
R E=\frac{\sum_{i=1}^{n} \frac{\mid \text { test }_{i}-\text { predicted }_{i} \mid}{\text { test }_{i}}}{n}
\end{gathered}
$$

用变异系数 (Coefficient of Variation, $C V$ ) 衡量数据离散程度:

$$
C V=\frac{\sqrt{\frac{1}{n} \sum_{i=1}^{n}\left(x_{i}-\bar{x}\right)^{2}}}{\bar{x}}
$$

\section{3 结果与验证}

\subsection{BP 神经网络隐含层节点数确定结果}

基于 3000 组 HydroLight 模拟数据,构建若干不同隐含层节点的 BP 神经网络分别进行训练, 训练性能 如图 4 所示, 随着节点数增加, $M S E$ 先减小后增大, 其最小值 (0.0306) 对应隐含层节点数为 10 , 所以本文采 用 10 个隐含层节点结构的 BP 神经网络. 


\subsection{HydroLight 模拟数据测试结果}

为了进一步验证本模型估算效果,选用 200 组 HydroLight 独立模拟数据作为神经网络的测试集 (HydroLight 模拟数据库共 3200 组数据, 随机抽取 其中 3000 组用于训练神经网络, 剩下 200 组用于 测试网络), 测试结果见图 5.

200 组数据数值范围基本覆盖 HydroLight 模 拟设定范围, $h$ 的测试值和估算值的相关系数和相 对误差分别为 0.96 和 $13.99 \%, \sigma$ 的测试值和估算 值的相关系数和相对误差分别为 0.98 和 $10.18 \%$, $h$ 和相对应 $\sigma$ 的相对误差同时小于 $30 \%$ 的占总数 据量的 $83.5 \%$,说明该 BP 神经网络模型具有较好 的稳定性和精确性,利用该网络模型基本可以满 足估算藻类高斯垂向分布结构参数精度要求.

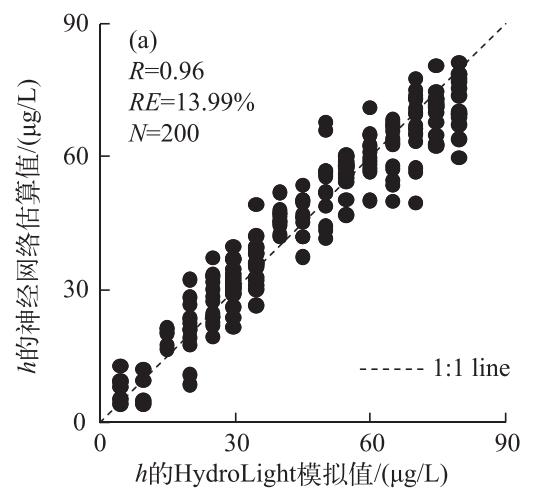

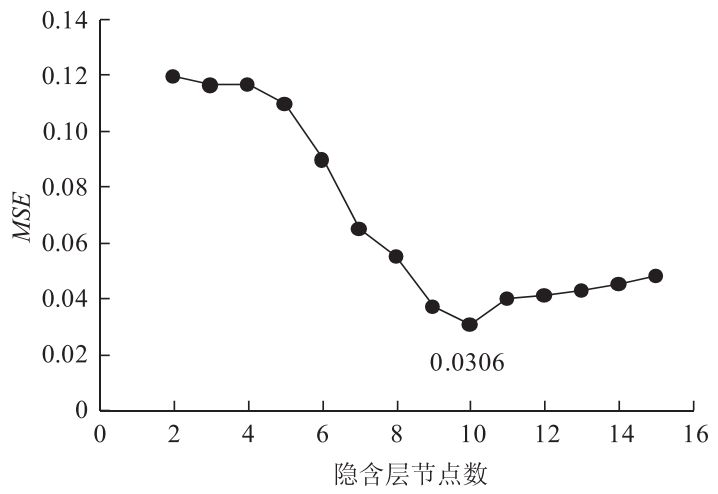

图 4 不同隐含层节点数对应的 $M S E$

Fig.4 Number of hidden layer nodes and corresponding MSE

图 5 HydroLight 模拟与网络估算的 $h$ 和 $\sigma$ 对比

Fig.5 Comparisons of simulated $h$ and $\sigma$ by HydroLight and test $h$ and $\sigma$

( $R$ represents the correlation coefficient, $R E$ represents the relative error, the same below)

\section{3 野外实测数据验证结果}

在模拟数据验证的基础上, 本研究利用野外实测数据对该神经网络模型的估算效果进行进一步验证, 探讨该模型对野外实测数据的适用性,验证结果如图 6 .

野外测量分别于 2013 年 5 月 28 日、 7 月 24 日和 2015 年 5 月 24 日进行,采样点位的分布覆盖了巢湖的 东湖区和西湖区, $h$ 和 $\sigma$ 的实测值和估算值的相关系数分别为 0.97 和 0.95 ,对应的相对误差分别为 $13.20 \%$ 和 $12.36 \%, h$ 和相对应 $\sigma$ 的相对误差同时小于 $30 \%$ 的占总数据量的 $87.5 \%$, 说明该 BP 神经网络针对野外实 测光谱数据, 依然具有较好地估算巢湖野外藻类高斯垂向分布结构的能力.

\section{4 讨论}

\subsection{HydroLight 辐射传输模拟的参数设定}

本文使用 HydroLight 进行辐射传输模拟时,太阳天顶角、风速、SPIM、 $a_{g}(440)$ 等都取 2014 年巢湖实测 数据的平均值, 为了检验辐射传输模拟结果对上述参数的敏感度, 本研究在假定叶绿素垂向分布结构不变 的情况下, 分别改变太阳天顶角、风速、SPIM 、 $a_{\mathrm{g}}(440)$, 探讨上述参数对 $R_{\mathrm{rs}}$ 模拟光谱的影响, 基于巢湖 2014 年数据, 具体取值范围分别为: $30^{\circ} \sim 60^{\circ} 、 1 \sim 5 \mathrm{~m} / \mathrm{s} 、 20 \sim 50 \mathrm{mg} / \mathrm{L} 、 0.16 \sim 1.16 \mathrm{~m}^{-1}$, 间隔分别为 $1^{\circ} 、 0.01 \mathrm{~m} / \mathrm{s} 、 1$ $\mathrm{mg} / \mathrm{L} 、 0.01 \mathrm{~m}^{-1}$. 结果 (图 7) 表明, 在 400 700 nm 波长范围内,太阳天顶角 $(C V \leqslant 0.66 \%$ )、风速 $(C V \leqslant 0.22 \%)$ 

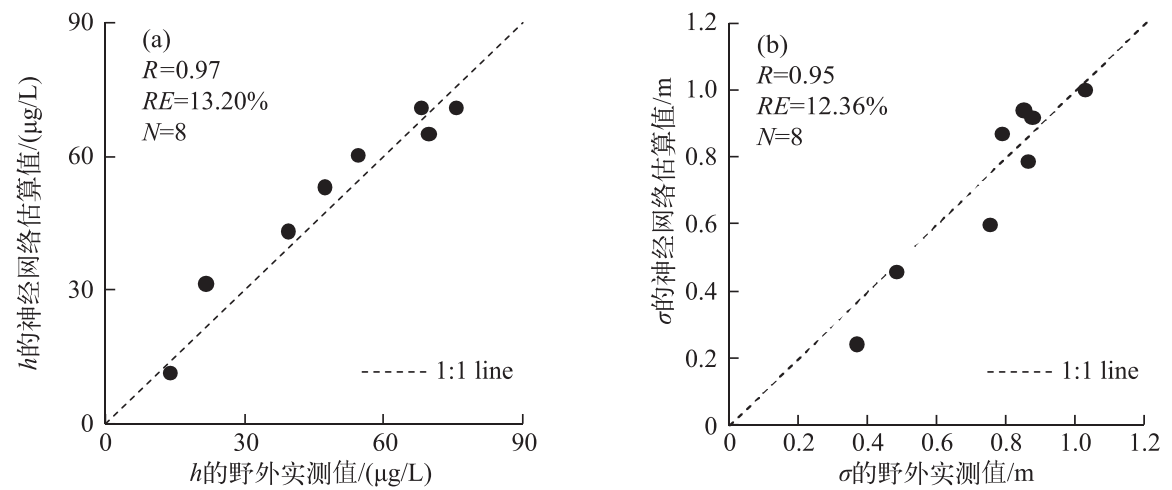

图 6 巢湖野外实测的与网络估算的 $h$ 和 $\sigma$ 对比

Fig.6 Comparisons of measured $h$ and $\sigma$ in Lake Chaohu and test $h$ and $\sigma$

对 $R_{\mathrm{rs}}$ 的影响不明显; $\mathrm{SPIM}$ 变化对 $R_{\mathrm{rs}}$ 的影响主要在 $550 \sim 700 \mathrm{~nm}$ 波段范围内 $(C V \geqslant 5.49 \%)$, 该范围内 $R_{\mathrm{rs}}$ 与 SPIM 呈正相关 $(R>0.9, P<0.01) ; a_{\mathrm{g}}(440)$ 变化对 $R_{\mathrm{rs}}$ 的影响则主要在绿光波段 $(C V \geqslant 1.46 \%)$, 变化规律与 SPIM 相反 $(R<-0.9, P<0.01)$.
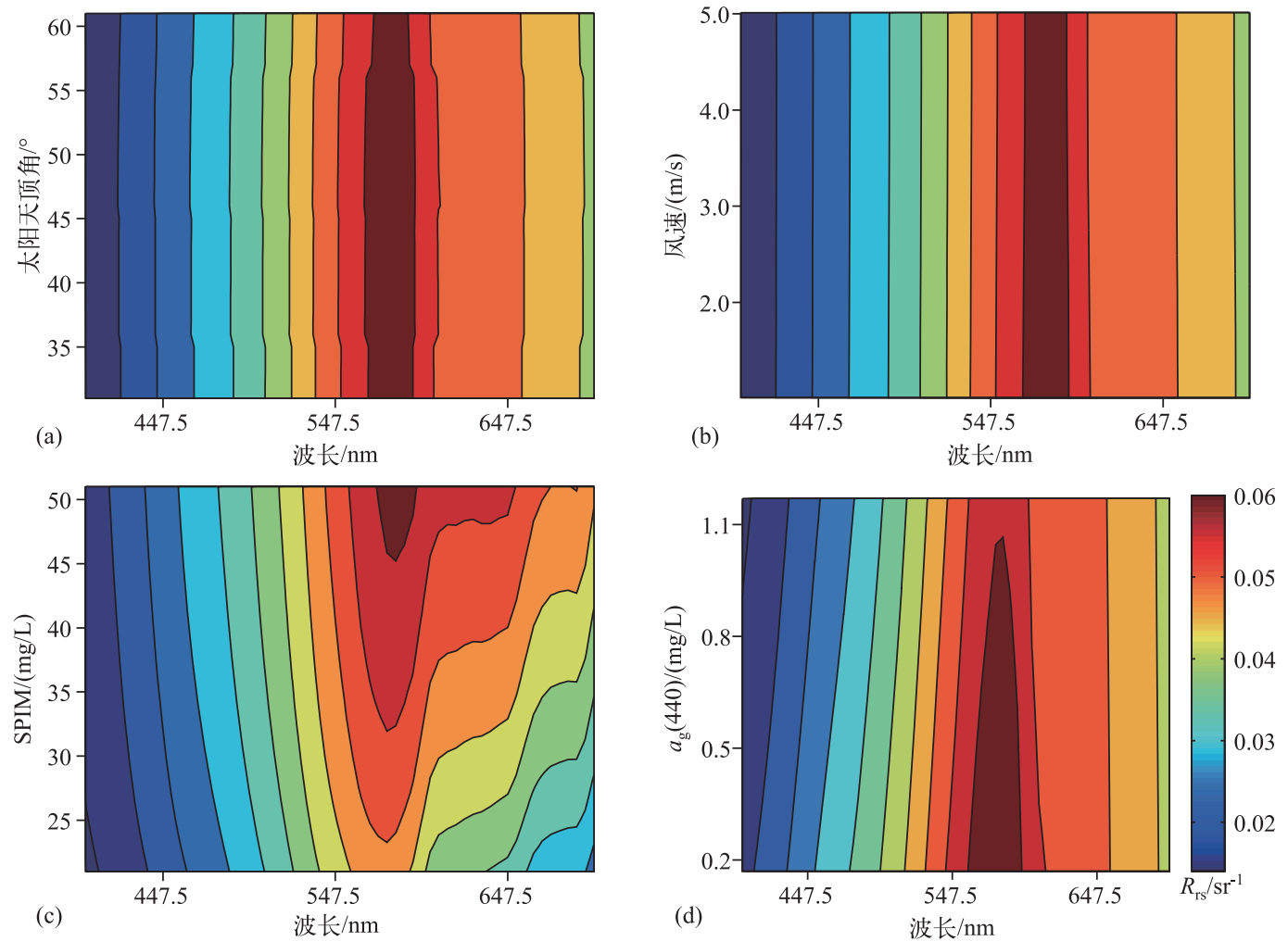

图 7 改变太阳天顶角、风速、SPIM、 $a_{\mathrm{g}}(440)$ 进行辐射传输模拟得到的 $R_{\mathrm{rs}}$ 等值线图

Fig.7 $R_{\mathrm{rs}}$ contour maps corresponding to varied solar zenith angle, wind speed, SPIM and $a_{\mathrm{g}}(440)$

从表 2 可以看出, 太阳天顶角、风速、SPIM、 $a_{\mathrm{g}}(440)$ 的改变对于 $R_{\mathrm{rs}}(469) 、 R_{\mathrm{rs}}(555) 、 R_{\mathrm{rs}}(645)$ 的影响总 体较小, $C V$ 都小于 $15 \%$. 此外, 巢湖叶绿素 a 浓度、SPIM、 $a_{\mathrm{g}}(440)$ 的平均值均呈现从西湖区到东湖区降低的 
趋势. 但是, 方差分析 (ANOVA) 结果表明叶绿素 a 浓度具有显著的空间差异 $(F=25.68, P<0.001)$, 而 SPIM $(P=0.35)$ 和 $a_{\mathrm{g}}(440)(P=0.24)$ 没有显著的空间差异. 说明本研究中构建的 BP 神经网络方法适用于太阳 天顶角、风速、SPIM 和 $a_{\mathrm{g}}(440)$ 变化的大规模数据集.

表 2 改变太阳天顶角、风速、SPIM $、 a_{\mathrm{g}}(440)$ 得到的 $R_{\mathrm{rs}}(469) 、 R_{\mathrm{rs}}(555) 、 R_{\mathrm{rs}}(645)$ 的变异系数 $(\%)$

Tab.2 CV (\%) of $R_{\mathrm{rs}}$ response to varied solar zenith angle, wind speed, SPIM and $a_{\mathrm{g}}(440)$

\begin{tabular}{ccccc}
\hline & 太阳天顶角 $\left(30 \sim 60^{\circ}\right)$ & 风速 $(1 \sim 5 \mathrm{~m} / \mathrm{s})$ & $\operatorname{SPIM}(20 \sim 50 \mathrm{mg} / \mathrm{L})$ & $a_{\mathrm{g}}(440)\left(0.16 \sim 1.16 \mathrm{~m}^{-1}\right)$ \\
\hline$R_{\mathrm{rs}}(469)$ & 0.59 & 0.14 & 6.19 & 5.65 \\
$R_{\mathrm{rs}}(555)$ & 0.63 & 0.17 & 5.49 & 2.67 \\
$R_{\mathrm{rs}}(645)$ & 0.62 & 0.21 & 13.01 & 0.61 \\
\hline
\end{tabular}

\section{2 影响藻类高斯垂向分布的主要因素}

巢湖水体中的浮游植物以蓝藻门、绿藻门和硅藻门为主 (一般达 $90 \%$ 以上), 其中又以蓝藻门居多 ${ }^{[33]}$. 巢湖中的绿藻门和硅藻门藻类主要以悬浮形态 (藻类在水体中运动形态主要包括悬浮、上浮和游泳) 存在, 其空间位置完全受水动力条件影响 ${ }^{[34]}$. 此外, 蓝藻有伪空泡/伪空胞气囊结构, 会根据其自身生理节律、生 理状态以及环境变化,通过上浮或者下沉选择最适宜的生长、生存空间 ${ }^{[35-36]}$.

图 8 是 BP 神经网络基于野外实测数据得到的叶绿素 a 浓度垂向结构和实测的叶绿素 a 浓度垂向数据. 4-9 月是蓝藻生物量增加的阶段 ${ }^{[12]}$, 所以 7 月 (图 8d) 叶绿素 a 浓度垂向整体比 5 月 (图 $8 \mathrm{a} \sim \mathrm{c}$ ) 高; 风浪的

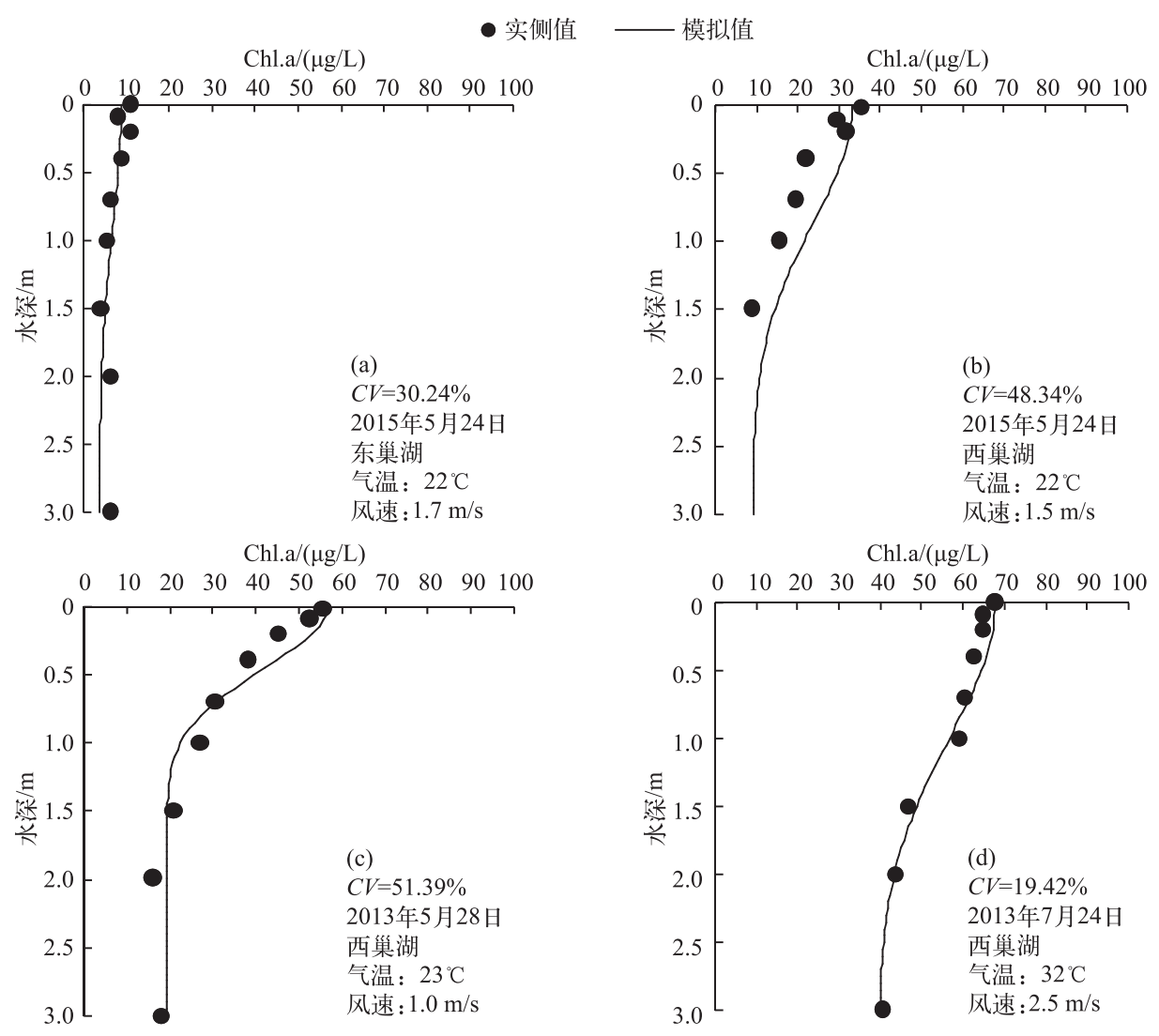

图 $8 \mathrm{BP}$ 神经网络输人实测 $R_{r s}$ 估算的与实测的叶绿素 $\mathrm{a}$ 浓度垂向分布

Fig.8 Vertical structures of simulated and measured chlorophyll-a concentrations 
摚动作用会促进水柱中藻颗粒上下混合、垂向分布趋于均匀 ${ }^{[37]}$, 风速越大, 叶绿素 $\mathrm{a}$ 浓度垂向变异系数越小 (图 8); 由于藻类生物活动与沉积物及水体中营养盐的相互作用, 巢湖西半湖 (图 8b) 叶绿素 a 浓度高于东 半湖 (图 8a $)^{[38]}$.

\section{$4.3 \mathrm{BP}$ 神经网络模型应用于卫星数据}

本研究构建 BP 神经网络过程中, 虽然选择输人参量仅考虑了 MODIS 卫星波段设置, 但是目前大多数 卫星(VIIRS、Landsat、HJ-1、EO-1、ALOS 等) 的波段均有上述波段或类似波段设置.

本文 $\mathrm{BP}$ 神经网络的输人参量使用的是 $R_{\mathrm{rs}}$, 欲将此模型应用于卫星数据上, 需要解决地面光谱与卫星 波段之间的波段响应函数关系以及大气校正的问题. 但是, 由于湖面上空气溶胶分布类型比海面更为复杂、 多变, II 类水体的大气校正成了国际水色遥感的难题 ${ }^{[16]}$. 如图 9 所示, 实测光谱根据 MODIS 光谱响应函数 重采样得到遥感反射率 $\left(R_{\mathrm{r} s \text { modis }}\right)$, 根据 SeaDAS 查找表模拟的不同气溶胶类型和厚度以及不同观测角度下 的 $R_{\mathrm{rs} \_ \text {modis }}$ 与经过瑞利校正和吸收气体校正的反射率 (Rayleigh Corrected Reflectance, $R_{\mathrm{rc} \_ \text {modis }}$ ) 的关系, 可以看 出不同气溶胶类型 $\left(R_{\mathrm{rc} \_ \text {modis }}(\lambda=469 \mathrm{~nm}, \mathrm{r} 75 \mathrm{f95} \mathrm{v} 01)-R_{\mathrm{rc} \_ \text {modis }}(\lambda=469 \mathrm{~nm}, r 80 \mathrm{f} 95 \mathrm{v} 01)=0.006\right)$ 和厚度 $\left(R_{\mathrm{rc} \_ \text {modis }}\right.$ $\left.\left(\lambda=469 \mathrm{~nm}, \tau_{\mathrm{a}}=1\right)-R_{\mathrm{rc} \_ \text {modis }}\left(\lambda=469 \mathrm{~nm}, \tau_{\mathrm{a}}=0.1\right)=0.132\right)$ 以及不同观测角度 $\left(R_{\mathrm{rc} \_ \text {modis }}\left(\lambda=469 \mathrm{~nm}, \theta_{0}=60^{\circ}\right.\right.$, $\left.\left.\theta=20^{\circ}, \varphi=40^{\circ}\right)-R_{\mathrm{rc} \_ \text {modis }}\left(\lambda=469 \mathrm{~nm}, \theta_{0}=40^{\circ}, \theta=40^{\circ}, \varphi=40^{\circ}\right)=0.004\right)$ 都会对遥感反射比造成不同影响, 且 对于不同波段的影响不同 $(\lambda=555 \mathrm{~nm}$ 时, 上述 3 个差值分别为 $0.004 、 0.119 、 0.007)$. 下一步研究中, 可以通 过寻找对上述大气影响不敏感的指数形式, 调整输人参数, 从而将模型推广应用至 MODIS 的 $R_{\mathrm{rc}}$ 数据 ${ }^{[39-40]}$.

此外, 应用卫星影像进行野外采样对应的遥感分析与高斯参数反演, MODIS 的象元尺度大于 $250 \mathrm{~m}$, 空 间分辨率较高的 Landsat 象元尺度是 $30 \mathrm{~m}$, 而湖泊水体水质变化的空间尺度往往小于 $10 \mathrm{~m}^{[1]}$, 导致在一个卫 星影像象元内, 可能存在多种藻类高斯垂向分布结构, 甚至存在多种垂向分布结构类型, 会降低估算高斯参 数的精确度.

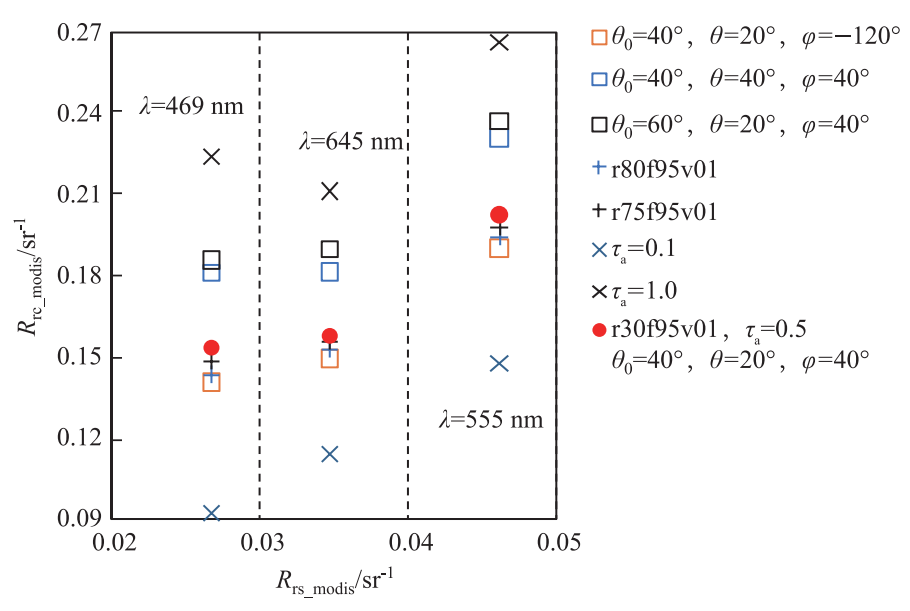

图 9 不同气溶胶类型、厚度与传感器观测角度下的 $R_{\mathrm{rc} \_ \text {modis }}$ 和 $R_{\mathrm{r} \_ \text {modis }}$

Fig.9 $R_{\text {rc_modis }}$ and $R_{\text {rs_modis }}$ under different atmospheric conditions and different sensor observation conditions

\subsection{Hydrolight 模拟与 BP 神经网络模型的局限性}

Hydrolight 模拟是在对自然水体的固有光学量进行参数化的基础上进行的理论模拟. 模拟得到的数据 集是一种概念化的结果, 与自然水体的测量数据会有一定差异. 由于野外仪器测量及室内分析本身存在误 差,理论上,不可能得到与自然水体的测量结果完全吻合的水体表观光学特性.

此外, 神经网络是一种黑箱模型, 通常都是 “不可解释” 的 ${ }^{[41]}$, 且神经网络隐含层节点数的确定缺乏严 格的理论依据指导 ${ }^{[30]}$. 上述不确定性均是该估算模型的主要误差来源. 


\section{5 结论}

本研究在分析高斯垂向分布结构参数对水体遥感反射比 $R_{\mathrm{rs}}$ 影响的基础上, 选取 $R_{\mathrm{rs}}(469) 、 R_{\mathrm{rs}}(555)$ 、 $R_{\mathrm{rs}}(645)$ 和 $C_{\text {Chl.a }}(0)$ 作为输人参量, 构建了估算高斯垂向结构参数 $\left(c_{0} 、 h\right.$ 和 $\left.\sigma\right)$ 的 BP 神经网络模型. HydroLight 模拟数据验证表明, $h$ 和 $\sigma$ 的 HydroLight 模拟值和网络估算值的相关系数分别为 0.96 和 0.98 , 对应的相 对误差分别为 $13.99 \%$ 和 $10.18 \%, h$ 和相对应 $\sigma$ 的相对误差同时小于 $30 \%$ 的占总数据量的 $83.5 \%$; 野外实测 数据验证表明, $h$ 和 $\sigma$ 的实测值和网络估算值的相关系数分别为 0.97 和 0.95 , 对应的相对误差分别为 $13.20 \%$ 和 $12.36 \%, h$ 和相对应 $\sigma$ 的相对误差同时小于 $30 \%$ 的占总数据量的 $87.5 \%$, 说明该 BP 神经网络能 够较好地估算巢湖野外藻类高斯垂向分布结构, 且 BP 神经网络反演巢湖藻类高斯垂向分布结构参数的方 法适用于太阳天顶角、风速、SPIM 和 $a_{\mathrm{g}}(440)$ 变化的大规模数据集. 进一步的研究中, 欲将 BP 神经网络模型 应用于卫星数据,大气校正和空间尺度问题亟待解决.

致谢: 感谢中国科学院南京地理与湖泊研究所“湖泊一流域科学数据共享平台”提供数据支持, 感谢意大利锡 耶纳大学 Steven A.Loiselle 教授对本文英文摘要的修改和润色, 感谢薛坤、李晶、陶慜、吴静汇、曹志刚、沈明、 张壹萱等参与采样.

\section{6 参考文献}

[ 1 ] Ma Ronghua, Tang Junwu, Duan Hongtao et al. Progress in lake water color remote sensing. J Lake Sci, 2009, 21 (2) : 143-158. DOI: 10.18307/2009.0201. [马荣华, 唐军武, 段洪涛等. 湖泊水色遥感研究进展. 湖泊科学, 2009, 21 (2) : 143-158.]

[ 2 ] Zaneveld JR, Barnard A, Boss E. Theoretical derivation of the depth average of remotely sensed optical parameters. Optics Express, 2005, 13(22) : 9052-9061.DOI: 10.1364/OPEX.13.009052.

[ 3 ] Ma Ronghua, Zhang Yuchao, Duan Hongtao. The status and development of the non-traditional lake water color remote sensing. J Lake Sci, 2016, 28 (2) : 237-245. DOI: 10.18307/2016.0201. [马荣华, 张玉超, 段洪涛. 非传统湖泊水色 遥感的现状与发展. 湖泊科学, 2016, 28(2) : 237-245.]

[ 4 ] Lewis MR, Cullen JJ, Platt T. Phytoplankton and thermal structure in the upper ocean: Consequences of non-uniformity in chlorophyll profile. Journal of Geophysical Research Oceans, 1983, 88 ( C4 ): 2565-2570. DOI: 10. 1029/JC088iC04p02565.

[ 5 ] Uitz J, Claustre H, Morel A et al. Vertical distribution of phytoplankton communities in open ocean: An assessment based on surface chlorophyll. Journal of Geophysical Research Oceans, 2006, 111 (C8) :275-303. DOI:10.1029/2005JC003207.

[ 6 ] Pertami ND, Kanno S, Arthana IW. Study on the vertical distribution of chlorophyll in coastal ocean; development of vertical model function at western sumbawa sea. Revista Transporte Y Territorio, 2012, (1) : 128-132.

[ 7 ] Hidalgo-Gonzalez RM, Alvarez-Borrego S. Chlorophyll profiles and the water column structure in the Gulf of California. Oceanologica Acta, 2001 , 24(1) : 19-28.DOI: 10.1016/S0399-1784(00) 01126-9.

[ 8 ] Millán-Núñez R, Alvarez-Borrego S, Trees CC. Modeling the vertical distribution of chlorophyll in the California Current System. Journal of Geophysical Research: Oceans, 1997, 102(C4) : 8587-8595.DOI: 10.1029/97JC00079.

[ 9 ] Feng Chunjing, Zhao Chaofang. Study of the vertical distribution of chlorophyll in the ocean based on artificial neural networks. Periodical of Ocean University of China, 2004, 34(3): 497-505. [冯春晶, 赵朝方. 基于人工神经网络研究海 水中叶绿素浓度的垂直分布. 中国海洋大学学报: 自然科学版, 2004, 34(3) : 497-505.]

[10] Liu Bin. The Feasibility of deriving the vertical distribution of chlorophyll concentration from sea surface reflectance measurements in case 1 water[ Dissertation]. Qingdao: Ocean University of China, 2009. [ 刘斌. 一类水域中从海面反射比反 演叶绿素浓度垂直分布初探 [学位论文]. 青岛: 中国海洋大学, 2009. ]

[11] Cao HS, Kong FX, Luo LC et al. Effects of wind and wind-induced waves on vertical phytoplankton distribution and surface blooms of Microcystis aeruginosa in Lake Taihu. Journal of Freshwater Ecology, 2006, 21(2) : 231-238. DOI: 10. 1080/02705060.2006.9664991.

[12] Kong Fanxiang, Gao Guang. Hypothesis on cyanobacteria bloom-forming mechanism in large shallow eutrophic lakes. Acta Ecol Sin, 2005, (3) : 589-595. [孔繁翔, 高光. 大型浅水富营养化湖泊中蓝藻水华形成机理的思考. 生态学报, 
$2005,(3): 589-595$.

[13] Wang Sumin, Dou Hongshen eds. Lakes of China. Beijing: Science Press, 1998. [王苏民, 窦鸿身. 中国湖泊志. 北京: 科学出版社, 1998.]

[14] Xue K, Zhang Y, Duan H et al. A remote sensing approach to estimate vertical profile classes of phytoplankton in a eutrophic lake. Remote Sensing, 2015, 7(11): 14403-14427. DOI:10.3390/rs71114403.

[15] Ma Mengxiao, Zhang Yuchao, Qian Xin et al. Vertical distribution of water quality and its influence on underwater light field in Lake Chaohu. Environmental Sciences, 2014, 35(5) : 1698-1707. DOI:10.13227/j.hjkx.2014.05.010. [马孟香, 张玉超, 钱新等. 巢湖水体组分垂向分布特征及其对水下光场的影响. 环境科学, 2014, 35(5): 1698-1707.]

[16] Pan Delu, Ma Ronghua. Several key problems of lake water quality remote sensing. J Lake Sci, 2008, 20(2) : 139-144. DOI : 10.18307/2008.0201. [ 潘德炉, 马荣华. 湖泊水质遥感的几个关键问题. 湖泊科学, 2008, 20(2) : 139-144.]

[17] Liu Xin, Jiang Yu, Gao Junfeng. Pollution characteristics of heavy metals and the risk assessment for the surface sediments from Lake Chaohu and its main tributary rivers. J Lake Sci, 2016, 28(3) : 502-512. DOI:10.18307/2016.0305. [ 刘新, 蒋豫, 高俊峰等. 巢湖湖区及主要出人湖河流表层沉积物重金属污染特征及风险评价. 湖泊科学, 2016, 28(3): 502-512.]

[18] Zhang Min, Kong Fanxiang. The process, spatial and temporal distributions and mitigation strategies of the eutrophication of Lake Chaohu (1984-2013). J Lake Sci, 2015, 27(5) : 791-798. DOI:10.18307/2015.0505. [张民, 孔繁翔. 巢湖富 营养化的历程、空间分布与治理策略(1984-2013 年). 湖泊科学, 2015, 27(5) : 791-798.]

[19] Chen X, Yang X, Dong X et al. Nutrient dynamics linked to hydrological condition and anthropogenic nutrient loading in Chaohu Lake (southeast China). Hydrobiologia, 2011, 661 (1) :223-234. DOI:10.1007/s10750-010-0526-y.

[20] Ma Shuzhan, Pan Jizheng, Wu Xiaodong et al. Purification efficiencies of a multi-stage bypass constructed wetland for treating polluted water from Nanfei River, Chaohu Catchment. J Lake Sci, 2016, 28 (2) : 303-311. DOI: 10.18307/2016. 0209. [马书占, 潘继征, 吴晓东等. 旁路多级人工湿地对巢湖流域南淝河水的净化效果. 湖泊科学, 2016, 28(2): 303-311.]

[21] Zhang Y, Ma R, Zhang M et al. Fourteen-year record (2000-2013) of the spatial and temporal dynamics of floating algae blooms in Lake Chaohu, observed from time series of MODIS images. Remote Sensing, 2015 , 7 (8) : 10523-10542. DOI: $10.3390 /$ rs 70810523 .

[22] Ma R, Tang J, Dai J. Bio-optical model with optimal parameter suitable for Taihu Lake in water colour remote sensing. International Journal of Remote Sensing, 2006, 27 (19) : 4305-4328. DOI: 10.1080/01431160600857428.

[23] Mobley CD. Estimation of the remote-sensing reflectance from above-surface measurements. Applied Optics, 1999,38 (36) : 7442-7455. DOI: 10.1364/AO.38.007442.

[24] Mobley CD ed. Light and water: Radiative transfer in natural waters. Academic Press, 1994.

[25] Bricaud A, Morel A, Prieur L. Absorption by dissolved organic matter of the sea (yellow substance) in the UV and visible domains 1. Limnology \& Oceanography, 1981, 26(1) : 43-53.DOI: 10.4319/lo.1981.26.1.0043.

[26] Li Suju, Wu Qian, Wang Xuejun et al. Correlations between reflectance spectra and contents of chlorophyll-a in Chaohu Lake. J Lake Sci, 2002, 14(3): 228-234. DOI:10.18307/2002.0306. [ 李素菊, 吴倩, 王学军等. 巢湖浮游植物叶绿 素含量与反射光谱特征的关系. 湖泊科学, $2002,14(3): 228-234$. $]$

[27] Zhou Yi, Zhou Weiqi, Wang Shixin et al. Applications of remote sensing techniques to inland water quality monitoring. Advances in Water Science, 2004, 15(3) : 312-317. [ 周艺, 周伟奇, 王世新等. 遥感技术在内陆水体水质监测中的应 用. 水科学进展, $2004, \mathbf{1 5}(3): 312-317$. ]

[28] Feng Wei, Feng Xuezhi, Ma Ronghua. Research on correlations between chlorophyll concentration and reflectance spectral of Taihu Lake. Remot Sens Inf, 2007, (1) : 18-21. [冯伟, 冯学智, 马荣华. 太湖水体叶绿素浓度与反射光谱特征关 系的研究. 遥感信息, 2007, 2007, (1) : 18-21.]

[29] Yacobi YZ, Gitelson A, Mayo M. Remote sensing of chlorophyll in Lake Kinneret using high spectral-resolution radiometer and Landsat TM: Spectral features of reflectance and algorithm development. Journal of Plankton Research, 1995, 17 (11) : 2155-2173.DOI: 10.1093/plankt/17.11.2155.

[30] Fan Jiani, Wang Zhenlei, Qian Feng. Research progress structural design of hidden layer in BP artificial neural networks. Control Engineering of China, 2005, (S1) : 109-113. [范佳妮, 王振雷, 钱锋. BP 人工神经网络隐层结构设计的研 究进展. 控制工程, 2005, (S1) : 109-113.] 
[31] Hecht-Nielsen R. Kolmogorov's mapping neural network existence theorem: Proceedings of the international conference on Neural Networks. New York: IEEE Press, 1987, 3: 11-13.

[32] Hüsken M, Jin Y, Sendhoff B. Structure optimization of neural networks for evolutionary design optimization. Soft Computing, 2005, 9(1) : 21-28. DOI: 10.1007/s00500-003-0330-y.

[33] Cheng Fang. The study of eutrophication condition and aquatic community structure of Taihu Lake[Dissertation]. Suzhou: Suzhou University, 2010. [成芳. 太湖水体富营养化与水生生物群落结构的研究 [学位论文]. 苏州: 苏州大 学, 2010.]

[34] Hu Hongjun, Wei Yinxin eds. Freshwater algae in China: Systems, classification and ecology. Beijing: Science Press, 2006. [胡鸿钧, 魏印心. 中国淡水藻类——系统、分类及生态. 北京:科学出版社, 2006.]

[35] Cheng Huimin, Qiu Baosheng. Cyanobacterial gas vesicles and their regulation on the vertical distribution of cyanobacteria in water body. Plant Physiology Communications, 2006, 42(5): 974-980. [成慧敏, 邱保胜. 蓝藻的伪空泡及其对蓝 藻在水体中垂直分布的调节. 植物生理学报, 2006, 42(5): 974-980.]

[36] Deng Daogui, Xie Ping, Zhou Qiong et al. Diel vertical migration of Microcystis and crustacean zooplankton in Lake Chaohu. Ecologic Science, 2006, 25(1):8-12. [ 邓道贵, 谢平, 周琼等. 巢湖微囊藻和浮游甲壳动物昼夜垂直迁移的初 步研究. 生态科学, 2006, 25(1):8-12.]

[37] Zhu Yongchun, Cai Qiming. The dynamic research of the influence of wind field on the migration of algae in Taihu Lake. J Lake Sci , 1997, 9(2) : 152-158. DOI: 10.18307/1997.0210. [ 朱永春, 蔡启铭. 风场对藻类在太湖中迁移影响的动 力学研究. 湖泊科学, $1997,9(2): 152-158$. ]

[38 ] Li Kun, Xiao Pu. Temporal and spatial distribution of chlorophyll-a concentration and its relationships with TN, TP concentrations in Lake Chaohu. Journal of Biology, 2011, 28(1): 53-56. [李堮, 肖莆. 巢湖叶绿素 a 浓度的时空分布及 其与氮、磷浓度关系. 生物学杂志, 2011, 28(1): 53-56.]

[39] Qi L, Hu C, Duan H et al. A novel MERIS algorithm to derive cyanobacterial phycocyanin pigment concentrations in a eutrophic lake: Theoretical basis and practical considerations. Remote Sensing of Environment, 2014, 154(154) : 298-317. DOI: $10.1016 /$ j.rse.2014.08.026.

[40] Liang Q, Zhang Y, Ma R et al. A MODIS-based novel method to distinguish surface Cyanobacterial scums and aquatic macrophytes in Lake Taihu. Remote Sensing, 2017, 9(2): 133.

[41] Zhou Zhihua, Chen Shifu. Rule extraction from neural networks. Journal of Computer Research and Development, 2002,39 (4) : 398-405. [ 周志华, 陈世福. 神经网络规则抽取. 计算机研究与发展, 2002, 39(4) : 398-405.] 